\title{
Neoadjuvant Docetaxel plus Carboplatin Versus Epirubicin plus Cyclophosphamide Followed by Docetaxel in Triple-negative, Early-stage Breast Cancer (NeoCART): Results from a Multicenter, Randomized Controlled, Open-label Phase II Trial
}

\section{Liulu Zhang}

Guangdong Academy of Medical Sciences: Guangdong Provincial People's Hospital

\section{Zhiyong Wu}

Shantou Central Hospital

Jie Li

Sun Yat-sen University First Affiliated Hospital

Ying Lin

Sun Yat-sen University First Affiliated Hospital

Zhenzhen Liu

Henan Cancer Hospital

\section{Yin Cao}

Dongguan People's Hospital

\section{Gangling Zhang}

bao tou shi zhong xin yi yuan: Inner Mongolia Baotou City Central Hospital

Hong-Fei Gao

Guangdong Academy of Medical Sciences: Guangdong Provincial People's Hospital

\section{Mei Yang}

Guangdong Academy of Medical Sciences: Guangdong Provincial People's Hospital

\section{Ci-Qiu Yang}

Guangdong Academy of Medical Sciences: Guangdong Provincial People's Hospital

\section{Teng Zhu}

Guangdong Academy of Medical Sciences: Guangdong Provincial People's Hospital

\section{Min-Yi Cheng}

Guangdong Academy of Medical Sciences: Guangdong Provincial People's Hospital

Fei Ji

Guangdong Academy of Medical Sciences: Guangdong Provincial People's Hospital Jieqing Li

Guangdong Academy of Medical Sciences: Guangdong Provincial People's Hospital 


\section{Kun Wang ( $\square$ gzwangkun@126.com )}

Guangdong Provincial People's Hospital https://orcid.org/0000-0001-9851-7080

\section{Research}

Keywords: Triple-negative breast cancer, neoadjuvant chemotherapy, carboplatin

Posted Date: June 8th, 2021

DOl: https://doi.org/10.21203/rs.3.rs-585170/v1

License: (c) (1) This work is licensed under a Creative Commons Attribution 4.0 International License. Read Full License 


\section{Abstract}

Background: Previous studies have shown that the addition of carboplatin to neoadjuvant chemotherapy regimens improved the pathologic complete response ( $\mathrm{pCR}$ ) rate in patients suffering from triple-negative breast cancer (TNBC). However, no studies have assessed the effects of the combination of docetaxel and carboplatin without anthracycline with taxane- and anthracycline-based regimens.

Methods: The NeoCART study was designed as a multicenter, randomized controlled, open-label, phase 2 trial to assess the efficacy and safety of docetaxel combined with carboplatin with taxane-and anthracycline-based neoadjuvant chemotherapy in untreated stage II-III TNBC. All eligible patients were randomly assigned, at a 1:1 ratio, to the experimental $D C b$ group (docetaxel plus carboplatin for six cycles) or the EC-D group (epirubicin plus cyclophosphamide followed by docetaxel). In both groups, pCR (ypTO/is ypN0) was evaluated as the primary outcome.

Results: Between September 1, 2016, and December 31, 2019, 93 patients from 6 participating centers were randomly assigned, and 88 patients were evaluated for the primary end-point (44 patients in the DCb group and 44 patients in the EC-D group). In the primary end point analysis, 27 patients in the DCb group achieved a pCR $(61.4 \%, 95 \% \mathrm{Cl} 47.0-75.8)$, and 17 patients in the EC-D group achieved a pCR $(38.6 \%, 95 \%$ $\mathrm{Cl} 24.3-53.0$ ); with a difference of $22.8 \%$ (odds ratio $2.52,95 \% \mathrm{Cl} 2.4-43.1$; p non-inferiority=0.004), noninferiority was met, and the DCb regimen was confirmed as superior to the EC-D regimen ( $p=0.044$, superiority margin of $5 \%$ ). At the end of the 37 -month median follow-up period, overall survival and eventfree rates were equivalent in both groups. The grade $3 / 4$ adverse events in the $\mathrm{DCb}$ group included anemia (4.5\%), thrombocytopenia (2.3\%), neutropenia (2.3\%) and an increased alanine aminotransferase (ALT)/aspartate aminotransferase (AST) ratio (2.3\%).

Conclusions: Compared with the taxane- and anthracycline-based regimen, the pCR rate of DCb was higher in TNBC patients.

Trial registration: ClinicalTrials.gov, NCT03154749. Registered 12 May 2017 - Retrospectively registered, https://clinicaltrials.gov/

\section{Background}

Studies show that patients with TNBC have poor prognosis due to the lack of targeted therapy. Despite advances in the treatment for primary breast cancer, cytotoxic chemotherapy is still the therapeutic base for TNBC.

Neoadjuvant therapy is usually recommended for TNBC. The benefits of neoadjuvant therapy include reducing the tumor size for breast-conserving surgery, avoiding axillary lymph node dissection, making inoperable tumors operable, and obtaining an in vivo evaluation of the tumor's chemosensitivity. Taxaneand anthracycline-based neoadjuvant regimens have become a standard treatment for TNBC, leading to 
longer overall survival (OS) and event-free survival (EFS) in patients who achieve a pathologic complete response $(\mathrm{pCR})^{1}$. The main focus of researchers has consistently been to improve the pCR rate of TNBC.

Platinum attacks cancer cells by inducing double-stranded DNA breaks, and TNBC may be sensitive to platinum ${ }^{2}$. Previous studies have shown a significantly improved $\mathrm{pCR}$ rate in patients with TNBC after adding carboplatin to neoadjuvant chemotherapy regimens $\mathbf{s}^{3,4}$, . Due to the long-term cardiotoxicity caused by anthracycline, several studies have explored the efficacy of neoadjuvant taxanes plus carboplatin regimens in TNBC and achieved satisfactory pCR rates ${ }^{6,7}$. However, no studies have assessed the effects of the combination of docetaxel and carboplatin without anthracycline versus taxane- and anthracycline-based neoadjuvant regimens on the pCR rate.

The NeoCART study was designed to assess the efficacy and safety of docetaxel plus carboplatin versus epirubicin plus cyclophosphamide followed by docetaxel neoadjuvant chemotherapy in TNBC.

\section{Methods}

\section{Patient Population}

The following criteria were used to enroll patients: Eastern Cooperative Oncology Group (ECOG) performance status of $0-1$; age $\geq 18$ years, cytologically or histologically confirmed noninflammatory invasive TNBC; a clinical stage of II-III (T1 $\mathrm{cN} 1-2$ or T2-4NO-2) and previously untreated. The diagnosis of TNBC was performed in line with the College of American Pathologists guidelines and American Society of Clinical Oncology (ASCO). According to immunohistochemistry results, progesterone receptor (PR) and estrogen receptor (ER) positivity rates were less than $1 \%$, the immunohistochemistry score for human epidermal growth factor receptor type 2 (Her2) staining was 0 or $1+$, and fluorescence in situ hybridization was utilized that no Her2 gene amplification. Adequate hematology, renal and liver function, normal heart function and negative pregnancy tests were analyzed for females with reproductive potential. Imaging tools (MRI, mammography or ultrasound) were used to measure the largest tumor diameter upon which T staging was determined. Staging evaluation comprised CT scanning for chest and abdomen and/or abdominal sonography and radionuclide bone imaging.

Patient with metastatic disease or who had received anticancer treatment were not qualified. Patient with a history of malignancy at another site (except for basal cell or squamous cell carcinoma of the skin and cervical carcinoma in situ) that had been fully treated were qualified if no disease was present for more than five years.

\section{Study Procedures}

The NeoCART study was designed as a multicenter, parallel group randomized controlled, open-label, phase 2 trial. The treatment allocation list was created, and randomization was performed centrally at the leading research center of Guangdong Provincial People's Hospital, Guangdong Academy of Medical Sciences. All eligible patients were randomly assigned at a 1:1 ratio to the experimental DCb group 
(docetaxel (75 mg/m² administered intravenously every 3 weeks) plus carboplatin (AUC $6 \mathrm{mg} / \mathrm{mL}$ per min, intravenously every 3 weeks) for six cycles) or the EC-D group (epirubicin $\left(90 \mathrm{mg} / \mathrm{m}^{2}\right)$ plus cyclophosphamide $\left(600 \mathrm{mg} / \mathrm{m}^{2}\right)$, both administered intravenously every 3 weeks for four cycles, followed by docetaxel $\left(100 \mathrm{mg} / \mathrm{m}^{2}\right)$ administered intravenously every 3 weeks for four cycles). The treatment regimen is shown in Fig. 1. Granulocyte colony-stimulating factors (G-CSF) use for prophylaxis of febrile neutropenia was permitted according to the ASCO guideline ${ }^{8}$. The hematological and biochemical indexes were evaluated every cycle. The dose of chemotherapy agents were reduced due to certain adverse events.

After surgery, those who exhibited residual disease were treated with capecitabine $\left(1000-1250 \mathrm{mg} / \mathrm{m}^{2}\right)$ given two-times a day for 1-14 days and cycled every 21 days for a total of 6-8 cycles ${ }^{9}$.

At 3 and 8 weeks after the last neoadjuvant chemotherapy treatment, definitive surgery was performed. The type of breast surgery (mastectomy or breast-conserving surgery) and axillary treatment (sentinel lymph node biopsy or axillary lymph node dissection) were determined by the treating surgeon.

The pCR rate, which was defined as the absence of invasive tumor cells in the breast and axilla (ypT0/is ypN0), was measured as the primary end-point. The PCR rate was determined by a local pathologist.

Secondary endpoints included EFS and OS assessed by an investigator, breast-conserving surgery rates and treatment-related toxicities were also examined. The National Cancer Institute Common Terminology Criteria for Adverse Events (version 4.0) was employed to assess the level of toxicity.

\section{Data Collection and Analysis}

Each number of subjects was randomly assigned to each group. Patients were randomized by means of a permuted block randomization scheme using an interactive response system (IxRS). Intention-to-treat methods were used and included in the analysis of all patients who started treatment.

The primary efficacy analysis was a non-inferiority comparison of the $\mathrm{pCR}$ rate between the DCb group and the EC-D group, using of a prespecified non-inferiority margin of $-5 \%$ as the absolute difference between the two groups. If non-inferiority was met, the superiority of the DCb regimen was to be tested ${ }^{10}$. Based on the findings of previous studies ${ }^{6}$, we assumed that $30 \%$ of the patients in the EC-D group and $55 \%$ of the patients in DCb group would achieve a pCR. With these assumptions, we estimated that we would need to enroll 82 patients (randomized 1:1) considering a one-sided a value of 0.025 for the hypotheses with $80 \%$ power.

The final date of data acquisition for this study was December 31, 2019. Data were analyzed with SPSS statistics version 22 (IBM Corporation). This study was registered at ClinicalTrials.gov (number NCT03154749).

\section{Study Approval}


The study was approved by the Research Ethics Committee of Guangdong Provincial People's Hospital, Guangdong Academy of Medical Sciences, and the protocol was reviewed by the responsible ethics committee is each center. This study conformed to the Declaration of Helsinki. All participants provided written informed consent.

\section{Results}

\section{Patient Characteristics}

Between September 1, 2016, and December 31, 2019, 93 patients from 6 participating centers in China were randomly assigned ( 47 patients to the DCb group and 46 patients to the EC-D group). The intentionto-treat analysis was performed for all participants except five who did not agree to receive treatment. Thus, 88 patients were evaluated for the primary end-point (44 in the DCb group and 44 in the EC-D group). Figure 2 shows the consort study flowchart.

The demographic information of 88 subjects who received treatment was presented in Table 1. Patient and treatment features were balanced in both groups. The median age of the 88 patients was 50 years (range $19-69$ years). In total, $1.1 \%, 35.3 \%$, and $63.6 \%$ of patients had histological grades of I, II, and III, respectively. A total of $76.1 \%$ of patients had $\mathrm{T} 1$ or $\mathrm{T} 2$ tumors, and $56.8 \%$ of the axillary lymph nodes were positive. Among the 88 patients, 41 received a germline BRCA status test, and $5(12.2 \%)$ had deleterious mutations. In the clinical evaluation before operation, 1 patient (2.3\%) in the DCb group had stable disease (SD) and 1 patient $(2.3 \%)$ had progressive disease; 4 patients $(9.0 \%)$ in the EC-D group had SD. The proportions of breast-conserving surgery in the DCb and EC-D groups were $36.4 \%$ and $38.6 \%$, respectively $(p=0.826)$. 
Table 1

Patient and Treatment Characteristics

\begin{tabular}{|c|c|c|c|c|}
\hline Characteristic, n (\%) & Total, $\mathbf{N}=\mathbf{8 8}$ & $\mathrm{DCb}, N=44$ & $E C-D, N=44$ & $p$ value \\
\hline Age, median (Q1, Q3), years & $50(39,57)$ & $50(38,59)$ & $49(40,56)$ & 0.844 \\
\hline \multicolumn{5}{|l|}{ Age } \\
\hline$<40$ years & $23(26.1)$ & $14(31.8)$ & $9(20.5)$ & \multirow[t]{2}{*}{0.225} \\
\hline$\geq 40$ years & $65(73.9)$ & $30(68.2)$ & $35(79.5)$ & \\
\hline \multicolumn{5}{|l|}{ Tumor Grade } \\
\hline I/II & $32(36.4)$ & $15(34.1)$ & $17(38.6)$ & \multirow[t]{2}{*}{0.658} \\
\hline III & $56(63.6)$ & $29(65.9)$ & $27(61.4)$ & \\
\hline \multicolumn{5}{|l|}{ Tumor size* } \\
\hline $\mathrm{T} 1 / \mathrm{T} 2$ & $67(76.1)$ & $33(75.0)$ & $34(77.3)$ & \multirow[t]{2}{*}{0.803} \\
\hline T3/T4 & $21(23.9)$ & $11(25.0)$ & $10(22.7)$ & \\
\hline \multicolumn{5}{|l|}{ Nodal involvement } \\
\hline Positive & $51(58.0)$ & $25(56.8)$ & $26(59.1)$ & \multirow[t]{2}{*}{0.829} \\
\hline Negative & $37(42.0)$ & $19(43.2)$ & $18(40.9)$ & \\
\hline \multicolumn{5}{|l|}{ Clinical Stage } \\
\hline II & $61(69.3)$ & $30(68.2)$ & $31(70.5)$ & \multirow[t]{2}{*}{0.817} \\
\hline III & $27(30.7)$ & $14(31.8)$ & $13(29.5)$ & \\
\hline \multicolumn{5}{|l|}{ Germline BRCA status } \\
\hline Deleterious mutation & $5(5.7)$ & $3(6.8)$ & $2(4.5)$ & \multirow[t]{2}{*}{0.686} \\
\hline No deleterious mutation & $36(40.9)$ & $16(36.4)$ & $20(45.5)$ & \\
\hline Not detected & $47(53.4)$ & $25(56.8)$ & $22(50)$ & \\
\hline \multicolumn{5}{|l|}{ Clinical response } \\
\hline $\mathrm{CR}$ & $19(21.6)$ & $10(22.7)$ & $9(20.5)$ & \multirow[t]{3}{*}{0.639} \\
\hline PR & $63(71.6)$ & $32(72.7)$ & $31(70.5)$ & \\
\hline SD & $5(5.7)$ & $1(2.3)$ & $4(9.0)$ & \\
\hline
\end{tabular}

Data are represented as $\mathrm{n}(\%)$ unless otherwise stated.

*Tumor size was assessed by the investigator by physical examination or imaging according to their discretion. 


\begin{tabular}{|c|c|c|c|c|}
\hline Characteristic, n (\%) & Total, $\mathbf{N}=\mathbf{8 8}$ & $\mathrm{DCb}, \mathrm{N}=\mathbf{4 4}$ & $E C-D, N=44$ & $p$ value \\
\hline PD & $1(1.1)$ & $1(2.3)$ & - & \\
\hline \multicolumn{5}{|c|}{ Breast conserving surgery } \\
\hline No & $55(62.5)$ & $28(63.6)$ & $27(61.4)$ & \multirow[t]{2}{*}{0.826} \\
\hline Yes & $33(37.5)$ & $16(36.4)$ & $17(38.6)$ & \\
\hline \multicolumn{5}{|c|}{ Data are represented as $\mathrm{n}(\%)$ unless otherwise stated. } \\
\hline
\end{tabular}

\section{Clinical Efficacy}

In the primary end point analysis, 27 patients in the DCb group achieved a pCR $(61.4 \%, 95 \% \mathrm{Cl} 47.0-75.8)$, and 17 patients in the EC-D group achieved a pCR (38.6\%, 95\% Cl 24.3-53.0); with a difference of $22.8 \%$ (odds ratio $2.52,95 \% \mathrm{Cl} 2.4-43.1 ; p$ non-inferiority $=0.004$ ), non-inferiority was met, and the $\mathrm{DCb}$ regimen was confirmed as superior to the EC-D regimen ( $p=0.044$, superiority margin of $5 \%$ )(Fig. 3 ). The pCR rate in five subjects who experienced detrimental germline BRCA mutations was $33.3 \%(1 / 3)$ in the DCb group and $100 \%$ in the EC-D group $(p=0.400)$.

A clinically relevant subgroup analysis was performed, and Fig. 4 shows the effect of the two treatment regimens on the proportion of patients who achieved a PCR. Regarding the different disease stages, the pCR rates of the DCb and EC-D groups were 73.3\% (22/30) and 48.4\% (15/31) in stage II, respectively $(95 \% \mathrm{Cl} 1.2-48.6 ; p=0.046)$, and $35.7 \%(5 / 14)$ and $15.4 \%(2 / 13)$ in stage III, respectively ( $95 \% \mathrm{Cl}-11.5$ $52.2 ; p=0.384)$.

\section{Survival analysis}

At the end of a 37 months median follow-up period, (range: 11-52 months, IQR: 29-44 months), EFS and OS appeared similar in the two groups: 3-year EFS rate $90.8 \%(95 \% \mathrm{Cl} 82.6-99.8 \%)$ for DCb, $88.3 \%(79.2-$ 98.5\%) for EC-D (hazard ratio 0.76, 95\% Cl 0.2-2.84, $\mathrm{P}=0.683$ ) (Fig. 5A). Three-year OS rates were $92.8 \%$ (85.3-100\%) for treated with DCb compared with 93.1\% (85.8-100\%) when treated with EC-D (hazard ratio $0.96,95 \% \mathrm{Cl} 0.19-4.76, \mathrm{P}=959)$ (Fig. $5 \mathrm{~B}$ ).

Patients achieving pCR showed markedly higher EFS and OS as relative to subjects with residual disease. Three-year EFS was $97.7 \%$ (95\% Cl: 93.4-100\%) and 81.2\% (95\% Cl: 70.3-93.9\%) in patients with and without pCR (log-rank P = 0.013) (Fig. 5C) and 3-year OS was 100\% and 85.6\% (75.5-97.1\%) for patients with and without $\mathrm{pCR}$, respectively (log-rank $\mathrm{P}=0.012$ ) (Fig. 5D).

\section{Treatment Delivery and Toxicity}

In total, $88.6 \%$ (39 of 44) and 97.7\% (43 of 44) of patients in the DCb and EC-D groups, respectively, completed the scheduled treatment. Seven $(15.9 \%)$ of 44 patients needed a decrease in carboplatin 
dosing from the initial dose of AUC 6 to AUC 5, and 1 patient (2.3\%) needed a second reduction to AUC 4.

The DCb protocol increased the total incidence of thrombocytopenia (20.5\%). The incidences of other adverse events were comparable between the two groups (Table 2). In DCb group, grade 3/4 adverse events included anemia (4.5\%), thrombocytopenia (2.3\%), neutropenia (2.3\%), an increased ALT/ AST ratio $(2.3 \%)$, diarrhea $(4.5 \%)$ and vomiting $(4.5 \%)$. The prevalence of thrombocytopenia (grade $3 / 4$ ) was similar in both groups, but grade $3 / 4$ neutropenia was more frequent in the EC-D group.

Table 2

Treatment-related Toxicities

\begin{tabular}{|c|c|c|c|c|}
\hline & \multicolumn{2}{|l|}{ Any grade } & \multicolumn{2}{|l|}{ Grade $\geq 3$} \\
\hline & DCb, $N=44$ & $E C-D, N=44$ & $\mathrm{DCb}, \mathrm{N}=44$ & $E C-D, N=44$ \\
\hline Anemia & $19(43.2)$ & $18(40.9)$ & $2(4.5)$ & - \\
\hline Thrombocytopenia & $9(20.5)$ & $1(2.3)$ & $1(2.3)$ & $1(2.3)$ \\
\hline Neutropenia & $14(31.8)$ & $9(20.5)$ & $1(2.3)$ & $4(9.1)$ \\
\hline ALT/AST* increased & $11(25.0)$ & $8(18.2)$ & $1(2.3)$ & - \\
\hline Constipation & $11(25.0)$ & $10(22.7)$ & - & - \\
\hline Diarrhea & $12(27.3)$ & $10(22.7)$ & $2(4.5)$ & - \\
\hline Nausea & $16(36.4)$ & $14(31.8)$ & - & - \\
\hline Vomiting & $13(29.5)$ & $11(25.0)$ & $2(4.5)$ & $3(6.8)$ \\
\hline Edema & $8(18.2)$ & $13(29.5)$ & - & - \\
\hline Fatigue & $16(36.4)$ & $19(43.2)$ & - & - \\
\hline Bone pain & $12(27.3)$ & $19(43.2)$ & - & $1(2.3)$ \\
\hline \multicolumn{5}{|c|}{ Data are represented as $\mathrm{n}(\%)$ unless otherwise stated. } \\
\hline
\end{tabular}

\section{Discussion}

Typically, TNBC is characterized by lack of Her2, PR, and ER. Due to the lack of other effective treatment, cytotoxic chemotherapy is the main therapy for TNBC. TNBC responds well to cytotoxic chemotherapy and has a higher pCR rate after neoadjuvant chemotherapy. Patients who obtained a pCR could achieve prolonged EFS and $\mathrm{OS}^{1}$. However, the clinical progression of TNBC is dismal relative to non-TNBC ${ }^{11-15}$. Therefore, the direction of current research is aimed at improving the pCR rate of neoadjuvant chemotherapy to improve the prognosis. 
Over time, taxane- and anthracycline-based neoadjuvant regimens have become a standard treatment for TNBC. Some phase 2 clinical trials have shown that adding platinum to taxane- and anthracycline-based neoadjuvant regimens can significantly improve the pCR rate. In a GeparSixto trial, more patients achieved a pCR rate of $53 \%$ in the group combining carboplatin with paclitaxel plus non-pegylated liposomal doxorubicin and bevacizumab relative to those treated without carboplatin ${ }^{4}$. In the CALGB 40603 trial, patients were randomly assigned to receive paclitaxel with or without carboplatin followed by doxorubicin plus cyclophosphamide. Similar to the GeparSixto trial results, the carboplatin-treated group achieved a higher pCR rate $(54 \% \text { vs } 41 \%, p=0.003)^{5}$. Sibylle Loibl et al. reported the results of the first randomized, double-blind, phase 3 trial, BrighTNess. The proportion of patients in the paclitaxel, carboplatin and veliparib group who achieved a pCR was higher than that in the paclitaxel only group (168 [53\%] of 316 patients vs. 49 [31\%] of 158 patients, $p<0.0001$ ), but the $p C R$ rate was similar to that of the paclitaxel plus carboplatin group (92 [58\%] of 160 patients, $p=0.36)^{3}$. The results of the BrighTNess study further established the important role of carboplatin in neoadjuvant chemotherapy for TNBC.

However, adding carboplatin to anthracycline plus paclitaxel substantially increased acute toxicity, with only $64 \%-88 \%$ of patients completing all treatment $\operatorname{cycles}^{3,4,5}$. Due to the long-term cardiotoxicity caused by anthracycline, several studies have explored the efficacy of neoadjuvant taxanes plus carboplatin regimens in TNBC and have achieved satisfactory $\mathrm{PCR}$ rates ${ }^{6,7}$. Previous results of our team showed that the pCR rate of 4 cycles of paclitaxel plus carboplatin in TNBC is $57.92 \%^{6}$. Priyanka Sharma et al. analyzed the efficacy of docetaxel plus carboplatin (DCb). Through combined analysis of two cohorts, they found that the total pCR rate and residual cancer burden (RCB) 0 / 1 rate of the $D C b$ regimen were $55 \%$ and $68 \%$, respectively ${ }^{7}$. The $\mathrm{pCR}$ rate of this $\mathrm{DCb}$ regimen seems to be numerically higher than that of the taxane- and anthracycline-based neoadjuvant regimens reported in several trials ${ }^{5,16,17}$. As a result, National Comprehensive Cancer Network (NCCN) guidelines have added weekly paclitaxel plus carboplatin or docetaxel plus carboplatin regimens for select patients with TNBC in the preoperative setting ${ }^{18}$. Recently published NeoSTOP study was confirmed that the $\mathrm{PCR}$ and RCB $0+1$ rates of carboplatin plus docetaxel $(\mathrm{CbD})$ were similar to carboplatin plus paclitaxel followed by doxorubicin plus cyclophosphamide ${ }^{19}$.

However, there is no study comparing the DCb regimen with the taxane- and anthracycline-based neoadjuvant regimens.

The NeoCART study is a multicenter prospective randomized controlled study to assess 6 cycles of DCb with epirubicin plus cyclophosphamide followed by docetaxel. Docetaxel combined with carboplatin showed a higher pCR rate (61.4\%) with an absolute difference in the pCR rate of $22.8 \%$. Our study supports the results of previous neoadjuvant chemotherapy studies on TNBC. In our study, the pCR rate was $38.6 \%$ in the control group, which is similar to the retes of $28 \%-46 \%$ reported in several trials of taxane- and anthracycline-based neoadjuvant regimens ${ }^{3,5,16,17}$. 
This multicenter, prospective, randomized controlled study showed that 6 cycles of DCb chemotherapy is tolerable and $88.6 \%$ of patients completed all 6 cycles of treatment. In the analysis of adverse reactions, the use of carboplatin resulted in increased thrombocytopenia, but most patients experienced grade $1 / 2$ adverse events; patients in the EC-D group may need a longer treatment time and are more likely to experience neutropenia.

A previous meta-analysis confirmed that patients with TNBC who achieved a pCR after neoadjuvant treatment had prolonged EFS and OS compared those who did not achieve a pCR ${ }^{1}$. According to the results of previous studies, the survival benefit of carboplatin is not clear. Our study showed that 6 cycles of DCb can significantly improve the possibility of achieving a pCR in patients with early TNBC and that the toxicity is controllable, while the rate of EFS and OS were similar to the taxane- and anthracyclinebased group.

In the BRCA status detection performed in our study, 5 patients $(12.2 \%)$ had deleterious mutations, which was consistent with the results of previous studies $3,7,20$. In theory, the DNA repair defects associated with BRCA mutation indicate that TNBC with BRCA mutation should be sensitive to platinum, a DNA damage drug. In the TNT study on advanced breast cancer, a subgroup analysis demonstrated that carboplatin had a higher the objective response rate than that of docetaxel $(68.0 \%$ vs. $33.3 \%, p=0.03)$ in the BRCA mutation subgroup, and progression-free survival was significantly different in the two groups (6.8 $\mathrm{m}$ vs. $4.4 \mathrm{~m}, \mathrm{p}=0.002)^{21}$. However, the results of neoadjuvant studies are not the same. The secondary analysis of the GeparSixto study showed that carboplatin did not further increase the pCR rate in patients with BRCA mutations ( $66.7 \%$ vs. $65.4 \%$ ), but it increased the $\mathrm{pCR}$ rate from $36.4-55 \%$ in patients with wild type $B R C A^{20}$. A subgroup analysis of the phase III BrighTNess study showed that carboplatin did not significantly increase the $\mathrm{pCR}$ rate in the population with BRCA mutations ${ }^{3}$. Similarly, Priyanka Sharma et al. found that the deleterious BRCA mutation was not associated with a PCR and RCB 0 or 1 , and similar pCR rates were found in patients with wild-type and mutant BRCA ${ }^{7}$. In addition, $40 \%-50 \%$ of BRCA wildtype TNBC had homologous recombination deficiency (HRD). Therefore, HRD may become a predictor of the benefits of platinum agents ${ }^{22,23,24}$. Further HRD detection and analysis in the NeoCART study is in progress.

Our study has some limitations. The control group of the NeoCART study, similar to the KEYNOTE-522 (NCT03036488) study and part of the BrighTNess study, used epirubicin plus cyclophosphamide followed by docetaxel every 3 weeks ${ }^{3}$. A recently published study from the Early Breast Cancer Trialists' Collaborative Group (EBCTCG) established the incremental benefit of 2-week regimens over 3-week regimens ${ }^{25}$. Additionally, the preferred recommended regimens in the NCCN guidelines were dose-dense doxorubicin/cyclophosphamide followed by weekly paclitaxel or every 2 weeks (ddAC-T). However, a head-to-head comparison is need between the EC-D and ddAC-T regimens for their effectiveness as neoadjuvant chemotherapy. The evidence for ddAC-T in the NCCN guidelines originates from the CALGB 9741 trial, which focuses on adjuvant chemotherapy ${ }^{26}$. In the EBCTCG meta-analysis, only $2583(6.9 \%)$ cases were included from neoadjuvant chemotherapy studies ${ }^{25}$. In addition, according to the results of 
the ECOG 1199 study, the efficacy of docetaxel administration every 3 weeks was the same as that of weekly paclitaxel ${ }^{27}$.

In summary, as assessed with the regimen of taxane- and anthracycline-based neoadjuvant therapy, docetaxel combined with carboplatin showed a higher pCR rate, a low incidence of controllable treatment-

related adverse events, and the similar survival rates. Docetaxel combined with carboplatin is an effective and promising neoadjuvant chemotherapy regimen for TNBC. The results need to be verified in a phase III study, and longer follow-up data are needed for the survival results.

\section{Conclusions}

Docetaxel plus carboplatin showed a higher PCR rate than that of the taxane-and anthracycline-based regimens. There was no significant difference in the breast-conserving rate, EFS and OS between the two treatment arms. In addition, docetaxel plus carboplatin had a low incidence of controllable treatmentrelated adverse events. This study provides an effective regimen option for neoadjuvant therapy of triplenegative breast cancer.

\section{Abbreviations}

TNBC: Triple-negative breast cancer; pCR: Pathologic complete response; ECOG: Eastern Cooperative Oncology Group; DCb: Docetaxel plus carboplatin; EC-D: Epirubicin plus cyclophosphamide followed by docetaxel; EFS: Event-free survival; OS: Overall survival; G-CSF: Granulocyte colony-stimulating factor; SD: Stable disease; ER: Estrogen receptor; PR: Progesterone receptor; Her2: Human epidermal growth factor 2; NCCN: National Comprehensive Cancer Network; RCB: residual cancer burden

\section{Declarations}

\section{Ethics approval and consent to participate}

The study was approved by the Research Ethics Committee of Guangdong General Hospital, Guangdong Academy of Medical Sciences (No. GDREC2016420H), and the protocol was reviewed by the responsible ethics committee at each participating site.

\section{Consent for publication}

Not applicable

\section{Availability of data and materials}

The datasets used and/or analysed during the current study are available from the corresponding author on reasonable request. 
The authors declare that they have no competing interests

\section{Funding}

This work was supported by the CSCO- constant Rui Tumor Research Fund区China (Y-HR2016-067); the National Natural Science Foundation of China (81871513); and the Science and Technology Planning Project of Guangzhou City(202002030236).

\section{Authors' contributions}

Conception and design: KW, LZ, ZW, JL

Provision of study materials or patients: LZ, ZW, YL, JL, ZL, YC, GZ, HFG, MY, CQY, TZ, MYC, FJ, JL, KW Collection and assembly of data: LZ, ZW, JL, HFG, MYC

Data analysis and interpretation: LZ, ZW, JL, KW

Manuscript writing: All authors

Final approval of manuscript: All authors

* The order of co-first authors is decided by the number of cases they contributed.

\section{Acknowledgements}

Not applicable

\section{References}

1. Cortazar P, Zhang L, Untch M, Mehta K, Costantino JP, Wolmark N, et al. Pathological complete response and long-term clinical benefit in breast cancer: the CTNeoBC pooled analysis. Lancet. 2014;384(9938):164-72.

2. Silver DP, Richardson AL, Eklund AC, Wang ZC, Szallasi Z, Li Q, et al. Efficacy of neoadjuvant Cisplatin in triple-negative breast cancer. J Clin Oncol. 2010;28(7):1145-53.

3. Loibl S, O'shaughnessy J, Untch M, Sikov WM, Rugo HS, Mckee MD, et al. Addition of the PARP inhibitor veliparib plus carboplatin or carboplatin alone to standard neoadjuvant chemotherapy in triple-negative breast cancer (BrighTNess): a randomised, phase 3 trial. Lancet Oncol. 2018;19(4):497-509.

4. Von Minckwitz G, Schneeweiss A, Loibl S, Salat C, Denkert C, Rezai M, et al. Neoadjuvant carboplatin in patients with triple-negative and HER2-positive early breast cancer (GeparSixto; GBG 66): a randomised phase 2 trial. Lancet Oncol. 2014;15(7):747-56. 
5. Sikov WM, Berry DA, Perou CM, Singh B, Cirrincione CT, Tolaney SM, et al. Impact of the addition of carboplatin and/or bevacizumab to neoadjuvant once-per-week paclitaxel followed by dose-dense doxorubicin and cyclophosphamide on pathologic complete response rates in stage II to III triplenegative breast cancer: CALGB 40603 (Alliance). J Clin Oncol. 2015;33(1):13-21.

6. Zhu T, Liu C, Zhang Y, Liu Y, Xu F, Zu J, et al. A phase II trial of dose-dense (biweekly) paclitaxel plus carboplatin as neoadjuvant chemotherapy for operable breast cancer. Breast Cancer Res Treat. 2016;156(1):117-24.

7. Sharma P, López-Tarruella S, García-Saenz JA, Ward C, Connor CS, Gómez HL, et al. Efficacy of neoadjuvant carboplatin plus docetaxel in triple-negative breast cancer: combined analysis of two cohorts. Clin Cancer Res. 2017;23(3):649-57.

8. Smith TJ, Bohlke K, Lyman GH, Carson KR, Crawford J, Cross SJ, et al. Recommendations for the use of WBC growth factors: American Society of Clinical Oncology clinical practice guideline update. $J$ Clin Oncol. 2015;33(28):3199-212.

9. Masuda N, Lee S-J, Ohtani S, Im Y-H, Lee E-S, Yokota I, et al. Adjuvant capecitabine for breast cancer after preoperative chemotherapy. N Engl J Med. 2017;376(22):2147-59.

10. Products CFPM. Points to consider on switching between superiority and non-inferiority. $\mathrm{Br} \mathrm{J}$ Clin Pharmacol. 2001;52(3):223-8.

11. Osborne C, Kannan L, Xie X, Ashfaq R, Bian A, Tripathy D. Neoadjuvant chemotherapy for basal-like breast cancer cohort: clinical and pathological outcomes. Breast Cancer Res Treat. 2006;100:53.

12. Dent R, Trudeau M, Pritchard KI, Hanna WM, Kahn HK, Sawka CA, et al. Triple-negative breast cancer: clinical features and patterns of recurrence. Clin Cancer Res. 2007;13(15):4429-34.

13. Carey LA, Dees EC, Sawyer L, Gatti L, Moore DT, Collichio F, et al. The triple negative paradox: primary tumor chemosensitivity of breast cancer subtypes. Clin Cancer Res. 2007;13(8):2329-34.

14. Liedtke C, Mazouni C, Hess KR, André F, Tordai A, Mejia JA, et al. Response to neoadjuvant therapy and long-term survival in patients with triple-negative breast cancer. J Clin Oncol. 2008;26(8):127581.

15. Kohler BA, Sherman RL, Howlader N, Jemal A, Ryerson AB, Henry KA, et al. Annual report to the nation on the status of cancer, 1975-2011, featuring incidence of breast cancer subtypes by race/ethnicity, poverty, and state. J Natl Cancer Inst. 2015; 107(6):36 - 8.

16. Arun B, Bayraktar S, Liu DD, Barrera AMG, Atchley D, Pusztai L, et al. Response to neoadjuvant systemic therapy for breast cancer in BRCA mutation carriers and noncarriers: a single-institution experience. J Clin Oncol. 2011;29(28):3739.

17. Esserman LJ, Berry DA, Demichele A, Carey L, Davis SE, Buxton M, et al. Pathologic complete response predicts recurrence-free survival more effectively by cancer subset: results from the I-SPY 1 TRIAL-CALGB 150007/150012, ACRIN 6657. J Clin Oncol. 2012;30(26):3242-9.

18. Gradishar WJ, Anderson BO, Abraham J, Aft R, Agnese D, Allison KH, et al. NCCN Clinical Practice Guidelines in Oncology (NCCN Guidelines ${ }^{\circledR}$ ). Breast Cancer. 2020;18(4):350-91. 
19. Sharma P, Kimler BF, O'dea A, Nye L, Wang YY, Yoder R, et al. Randomized Phase II Trial of Anthracycline-free and Anthracycline-containing Neoadjuvant Carboplatin Chemotherapy Regimens in Stage I-III Triple-negative Breast Cancer (NeoSTOP). Clin Cancer Res. 2021;27(4):975-82.

20. Hahnen E, Lederer B, Hauke J, Loibl S, Kröber S, Schneeweiss A, et al. Germline mutation status, pathological complete response, and disease-free survival in triple-negative breast cancer: secondary analysis of the GeparSixto randomized clinical trial. JAMA Oncol. 2017;3(10):1378-85.

21. Tutt A, Tovey H, Cheang MCU, Kernaghan S, Kilburn L, Gazinska P, et al. Carboplatin in BRCA1/2mutated and triple-negative breast cancer BRCAness subgroups: the TNT Trial. Nat Med. 2018;24(5):628-37.

22. Telli ML, Jensen KC, Vinayak S, Kurian AW, Lipson JA, Flaherty PJ, et al. Phase II study of gemcitabine, carboplatin, and iniparib as neoadjuvant therapy for triple-negative and BRCA1/2 mutation-associated breast cancer with assessment of a tumor-based measure of genomic instability: PrECOG 0105. J Clin Oncol. 2015;33(17):1895.

23. Isakoff SJ, Mayer EL, He L, Traina TA, Carey LA, Krag KJ, et al. TBCRC009: a multicenter phase II clinical trial of platinum monotherapy with biomarker assessment in metastatic triple-negative breast cancer. J Clin Oncol. 2015;33(17):1902-9.

24. Vollebergh MA, Lips EH, Nederlof PM, Wessels LF, Wesseling J, Vd Vijver MJ, et al. Genomic patterns resembling BRCA1-and BRCA2-mutated breast cancers predict benefit of intensified carboplatinbased chemotherapy. Breast Cancer Res. 2014;16(3):R47.

25. Gray R, Bradley R, Braybrooke J, Liu Z, Peto R, Davies L, et al. Increasing the dose intensity of chemotherapy by more frequent administration or sequential scheduling: a patient-level metaanalysis of 37298 women with early breast cancer in 26 randomised trials. Lancet. 2019;393(10179):1440-52.

26. Citron ML, Berry DA, Cirrincione C, Hudis C, Winer EP, Gradishar WJ, et al. Randomized trial of dosedense versus conventionally scheduled and sequential versus concurrent combination chemotherapy as postoperative adjuvant treatment of node-positive primary breast cancer: first report of Intergroup Trial C9741/Cancer and Leukemia Group B Trial 9741. J Clin Oncol. 2003;21(8):1431-9.

27. Sparano JA, Zhao F, Martino S, Ligibel JA, Perez EA, Saphner T, et al. Long-term follow-up of the E1199 phase III trial evaluating the role of taxane and schedule in operable breast cancer. J Clin Oncol. 2015;33(21):2353.

\section{Figures}




\section{Key Eligibility Criteria}

- Age $\geq 18$ years

- Newly diagnosed

TNBC of ether

T1cN1-2 or

T2-4N0-2

- ECOG PS 0 - 1
Q3W, 6 cycles

\section{Docetaxel $75 \mathrm{mg} / \mathrm{m}^{2}$}

Carboplatin AUC 6

\section{$\mathbf{R}$}

$1: 1$

Q3W, 8 cycles

Docetaxel $100 \mathrm{mg} / \mathrm{m}^{2}$
Epirubicin $90 \mathrm{mg} / \mathrm{m}^{2}$

Cyclophosphamide $600 \mathrm{mg} / \mathrm{m}^{2}$

\section{Figure 1}

Schema of randomized phase II NeoCART trial.

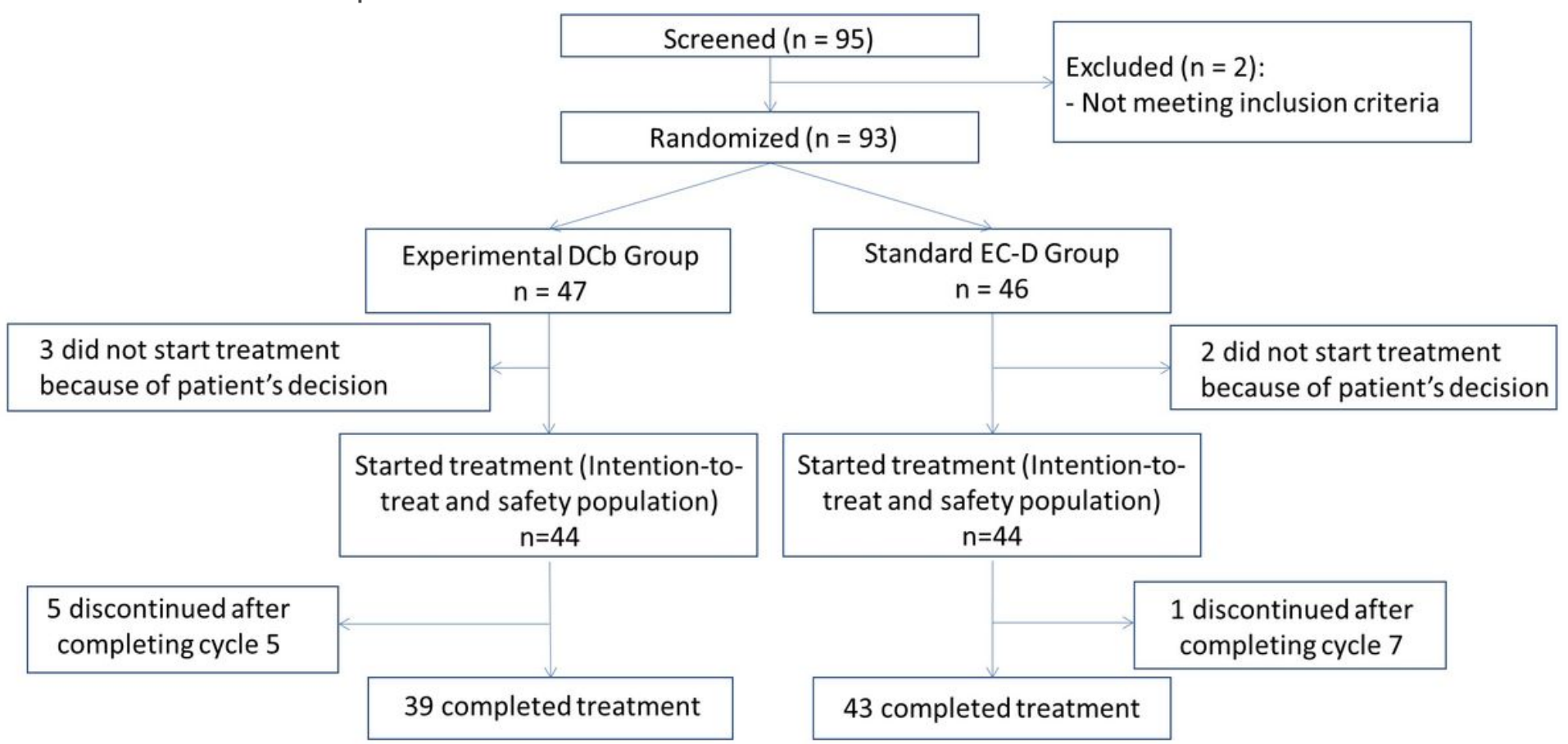

\section{Figure 2}

The consort study flowchart. DCb, docetaxel plus carboplatin; EC-D, epirubicin plus cyclophosphamide followed by docetaxel. 


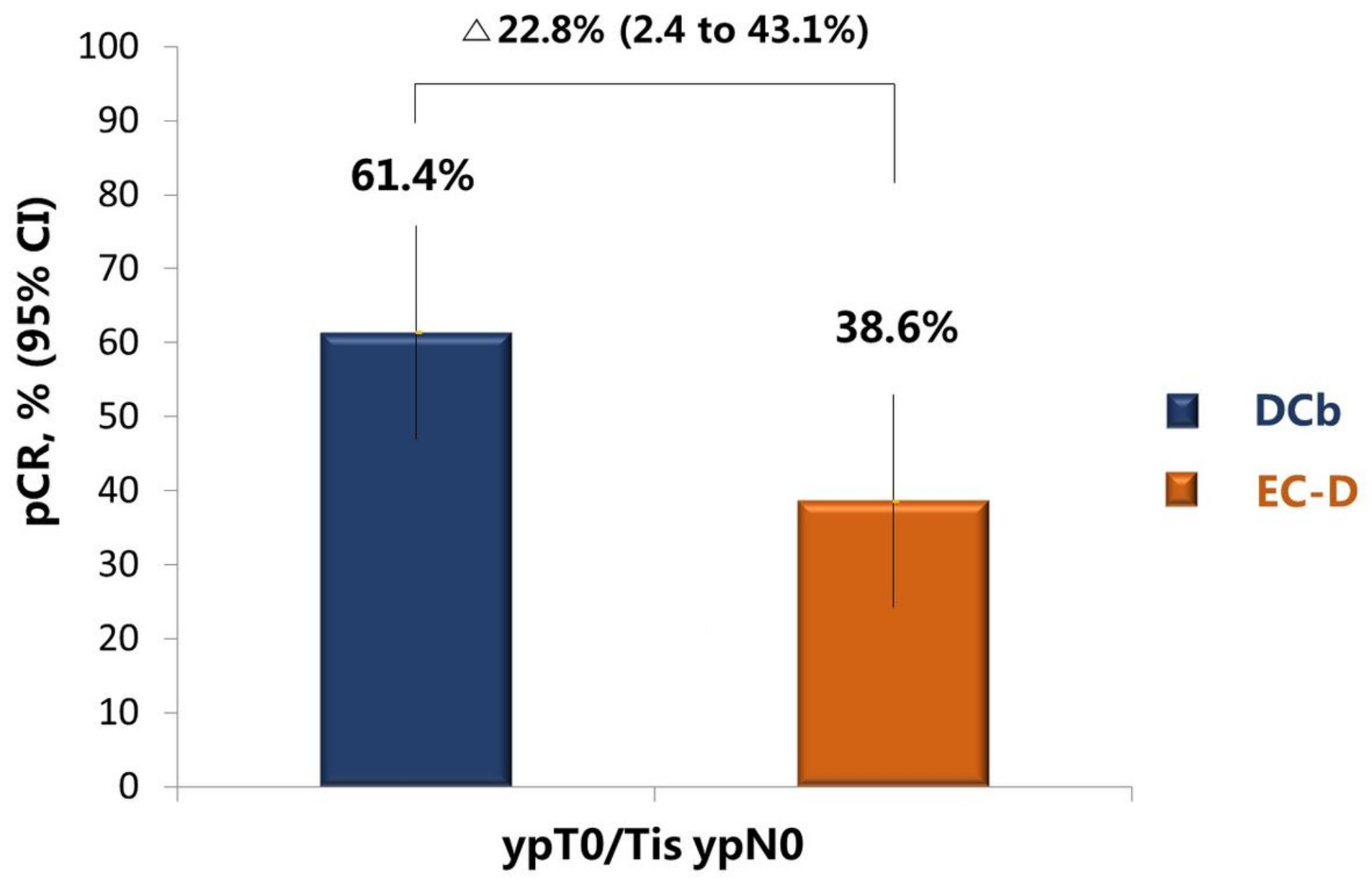

Figure 3

Pathologic complete response (pCR) of the breast/axilla (ypTo/isNo) 


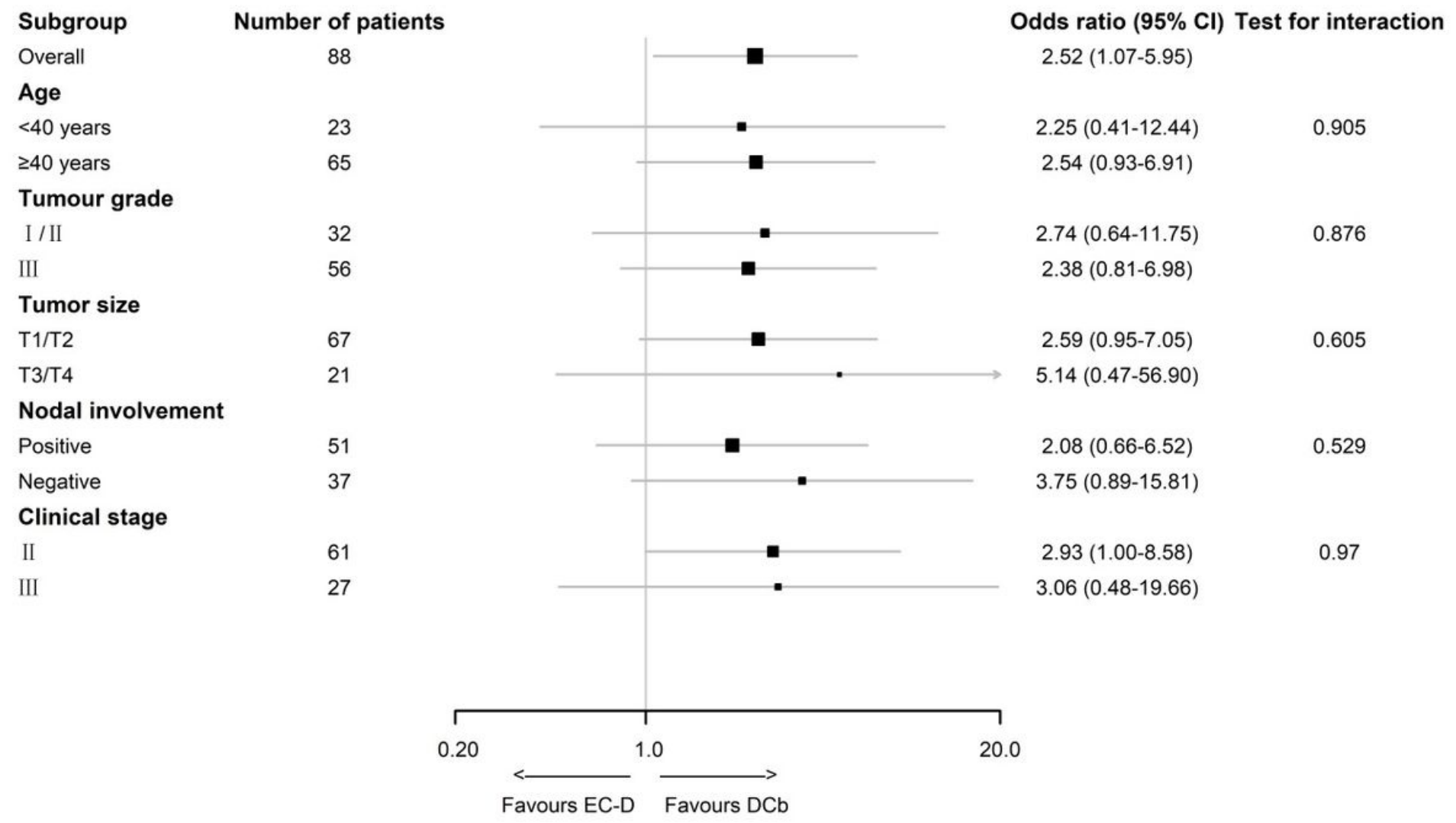

\section{Figure 4}

Effect of the two treatment regimens overall and in subgroups 


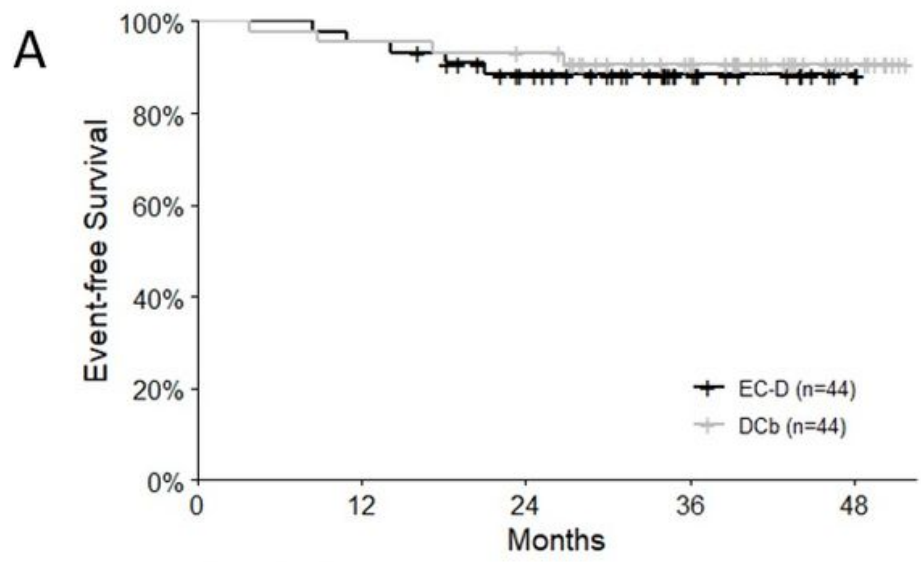

\section{No. at risk}

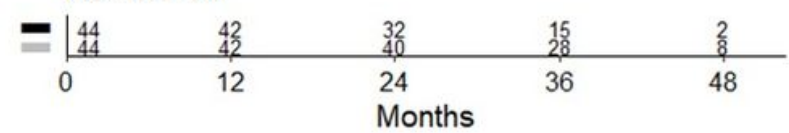

C

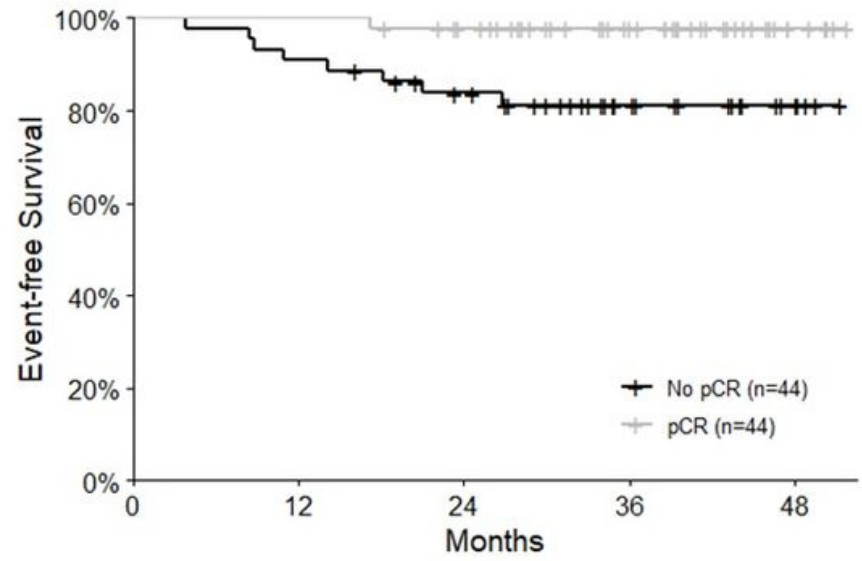

No. at risk

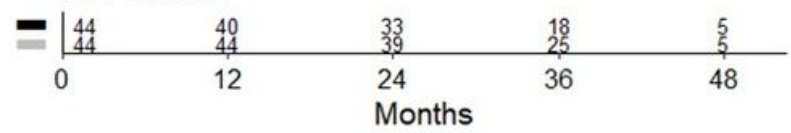

B

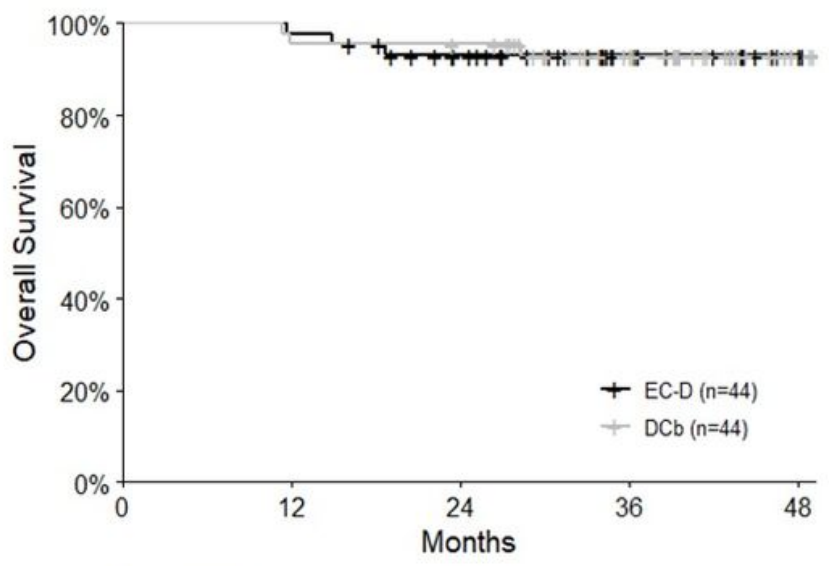

No. at risk
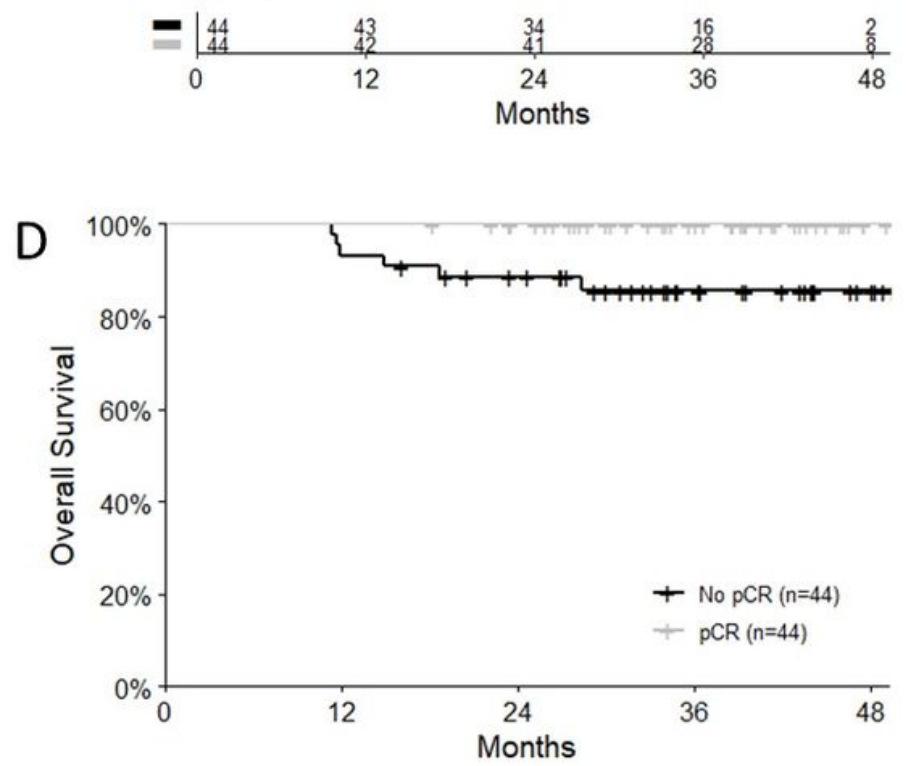

No. at risk

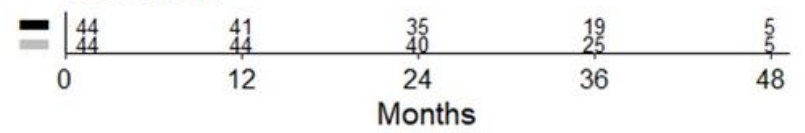

\section{Figure 5}

Event-free survival ( $A$ and $C$ ) and overall survival ( $B$ and $D$ ) according to treatment groups and $p C R$ status. *pCR, pathological complete response 